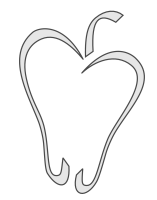

ISSN 1852-4249

\title{
Commentary on "Graphite and its hidden superconductivity"
}

\author{
E. M. Forgan ${ }^{1 *}$
}

I write this comment on the article by Esquinazi [1] as an expert on superconductivity but not as an expert on graphite. I should also mention that in 1986, a student asked me what I thought about a paper written by Bednorz \& Muller in Z. Phys. After looking at it carefully, I commented that it represented measurements on a mixed-phase sample, which had a resistivity $\sim 1000$ times that of copper at room temperature. The resistivity was increasing as the temperature was lowered, i.e., behaving in a non-metallic fashion. At $\sim 35 \mathrm{~K}$, the resistivity began to fall, but had not become "zero" until 10 K. Note that "zero" on the scale of the graph in the paper might just be the resistivity of copper at room temperature. Hence I concluded that there was no proof of superconductivity (such as the Meissner effect) and I highlighted the word "possible" in the title of the paper. However, other workers were more "gullible" and attempted to repeat and extend this work. It turned out that the phenomenon was very "democratic" and widely reproducible (unlike the equally surprising reports of "cold fusion" a few years later). So here I try to discuss whether the proposed superconductivity in graphite at elevated temperatures is real or not. One initial bibliographic comment may well be relevant: the papers reporting signs of superconductivity in graphite have a very restricted group of authors, suggesting that the phenomenon may

\footnotetext{
*E-mail: ted.forgan@gmail.com

1 School of Physics \& Astronomy, University of Birmingham, U.K.
}

not be "democratic". Some workers have become persuaded that the phenomenon is real, but they have not yet convinced a much wider audience, who probably feel that exceptional claims need exceptionally strong evidence.

It is clear from the discussion in section I of the paper, and a review of the extensive literature, that graphite is a complicated and sometimes irreproducible material. This is partly due to the weak interlayer forces, which mean that it does not always stack in an ideal ABAB hexagonal pattern. In addition, after the discovery of single-layer graphene, we know that independent layers may exist with extremely high mobility, conducting only in the basal plane direction. Even without this complication, graphite is a highly anisotropic material: this can easily cause difficulties in measuring transport properties, since the anisotropy in resistivity can give non-uniform current distributions. The effect of magnetic field on electron motion is also very anisotropic, with c-axis fields having strong effects on transport properties, and basal plane fields having almost no effect. Furthermore, the diamagnetic susceptibility is strong, very anisotropic and temperature-dependent. This bulk property and many others, such as the de Haas van Alphen effect in large samples [2] have been understood in general terms [3] as a consequence of a semi-metallic band-structure [4] since $\sim 1960$.

I now turn to the various sections of the paper. In section II, there is an account of strong magnetoresistance effects. Similar effects have also been observed in bismuth [5] and have a very interesting explanation [5] in terms of the semi-metallic prop- 
PAPers in Physics, vol. 5, ART. 050008 (2013) / E. M. Forgan

erties of graphite and bismuth, so there is no need to propose a superconducting explanation for this. In section III, a tiny hysteresis in magnetoresistance is described. Two comments are relevant here: the author notes that the sign of the hysteresis is opposite to that expected for a superconductor and limits himself to stating that the data provide "striking hints that granular superconductivity is at work in some regions of these samples". This is hardly definitive proof. Section IV is headed "Direct evidence for Josephson behavior". This quotes data from a recent publication from the author's group [6]. It is worth noting that these measurements were made with very small currents, so that the limit of measurement value is in the ohms region or greater. In some cases [6], apparent negative resistance values were observed. This can easily occur (and has been observed by myself) in a layered material. The phenomenon arises from nonuniform current flow enhanced by the resistivity anisotropy, combined with voltage leads which effectively make contact at different positions along the c-axis of the sample. It seems likely that these curious results, and their current-dependence, arise from non-ideal connections of the voltage and/or current leads. Other odd features of the results, such as sample-dependent noise at low temperatures, and the fact that magnetic fields could increase, decrease or have no effect on the voltages observed, also cast great doubt on the Josephson interpretation.

In section $\mathrm{V}$, we have an account of some magnetic susceptibility measurements, such as those reported in [7] on graphite "doped" with water. The hysteresis loops reported in that paper correspond to a maximum signal only $\lesssim 1 \%$ of the c-axis susceptibility of graphite. The value of this susceptibility, though relatively large, is $<0.001$ (SI dimensionless units). Hence if the width of the hysteresis loop observed in these measurements corresponds to a Meissner signal from superconductivity, then this supposed superconductivity occupies a volume fraction $\lesssim 10^{-5}$. Esquinazi et al. contend that this is consistent with superconductivity only present at somewhat ill-defined interfaces; however, it also means that one has to beware of artifacts. In response to [7], a colleague repeated their measurements as an undergraduate project [8]. Their clear conclusion was that if the correct diamagnetic background slope (that obtained at large fields) is sub- tracted, then the hysteresis corresponds to a tiny ferromagnetic component. However, if a slightly different background is chosen, the hysteresis loops look somewhat like the response of a granular superconductor. However, for a granular superconductor the hysteresis peaks should lie away from the vertical axis in the bottom right/top left corners (see e.g. [9]) and this is contrary to what is observed in graphite. Further evidence that this hysteresis is not due to superconductivity may be obtained from its temperature-dependence. We see in [7] that the hysteresis at $300 \mathrm{~K}$ is essentially the same as that at $5 \mathrm{~K}$. We bear in mind that by assumption the superconductivity is confined to an atomic layer, and that the higher the $T_{c}$ of a superconductor the shorter the coherence length. These two together ensure that thermal fluctuations (which are already very noticeable at $T \sim 100 \mathrm{~K}$ in cuprate materials) would be huge for any room temperature graphite superconductivity [10]. Thermal fluctuations would greatly reduce vortex pinning and magnetic irreversibility at room temperature, contrary to what is observed. On the other hand, a saturated ferromagnetic response would be almost temperature-independent for temperatures well below the Curie point. There are further measurements [11] which appear to show magnetic hysteresis (as a function of direction of temperature sweep, not as a function of field) going to zero at $400 \mathrm{~K}$. However, this temperature is where the sweep direction changes, so the hysteresis with temperature is by definition zero at $400 \mathrm{~K}$. Once again the differences in the magnetic signals are a tiny fraction of the total sample magnetization. There are many possible reasons (both real and due to experimental artifacts) why measurements on a sample taken on heating and cooling might disagree. Hence, the rather complicated results summarized in Esquinazi's paper cannot confidently be ascribed to (as yet not understood) superconducting effects.

I cannot give an overriding simple explanation for all the different results reported in Equinazi's paper, but neither can the author. In some cases this is because the proposed superconductivity is a "moving target": sometimes with a $T_{c} \sim 25 \mathrm{~K}$, and sometimes $T_{c}$ well above room temperature; sometimes superconducting effects are suppressed by magnetic field and sometimes enhanced at high fields. In interpreting the evidence presented, the 
Papers in Physics, vol. 5, ART. 050008 (2013) / E. M. Forgan

author has a tendency to jump to a superconducting interpretation, when others are perfectly possible. Unless and until graphite samples can be produced which exhibit the Meissner effect for a volume fraction of at least $1 \%$, and which show direct evidence of quantum coherence (hysteresis which might arise from Josephson networks or from other causes is not direct evidence), I expect that the scientific community at large will not accept that graphite exhibits high-temperature superconductivity.

[1] P Esquinazi, Graphite and its hidden superconductivity, Pap. Phys. 5, 050007 (2013).

[2] J W McClure, Band structure of graphite and de Haas-van Alphen effect, Phys. Rev. 108, 612 (1957).

[3] J W McClure, Theory of diamagnetism of graphite, Phys. Rev. 119, 606 (1960).

[4] J-C Charlier, X Gonze, J-P Michenaud, Firstprinciples study of the electronic properties of graphite, Phys. Rev. B 43, 4579 (1991).

[5] X Du, S W Tsai, D L Maslov, A F Hebard, Metal-insulator-like behavior in semimetallic bismuth and graphite, Phys. Rev. Lett. 94, 166601 (2005).
[6] A Ballestar, J Barzola-Quiquia, T Scheike, P Esquinazi, Josephson-coupled superconducting regions embedded at the interfaces of highly oriented pyrolytic graphite, New J. Phys. 15, 023024 (2013).

[7] T Scheike, W Bhlmann, $\mathrm{P}$ Esquinazi, J Barzola-Quiquia, A Ballestar, A Setzer, Can doping graphite trigger room temperature superconductivity? Evidence for granular hightemperature superconductivity in water-treated graphite powder, Adv. Mater. 24, 5826 (2012).

[8] M Robson, P Diwell (unpublished). Supervised by E Blackburn, School of Physics \& Astronomy, University of Birmingham, U.K. (2012).

[9] S Senoussi, C Aguillon, S Hadjoudj, The contribution of the intergrain currents to the low field hysteresis cycle of granular superconductors and the connection with the micro- and macrostructures, Physica C 175, 215 (1991).

[10] A Gurevich, Challenges and opportunities for applications on unconventional superconductors, Annu. Rev. Cond. Matter Phys., in press.

[11] T Scheike, P Esquinazi, A Setzer, W Böhlmann, Granular superconductivity at room temperature in bulk highly oriented pyrolytic graphite samples, Carbon 59, 140 (2013). 